\title{
Predicting Stereopsis in Macular Degeneration
}

\author{
Preeti Verghese and Saeideh Ghahghaei \\ Smith-Kettlewell Eye Research Institute, San Francisco, California 94115
}

Each of our eyes sees a slightly different view of the physical world. Disparity is the small difference in position of features in the retinal images; stereopsis is the percept of depth from disparity. A distance between corresponding features in the retinal images of the two eyes smaller than the "upper disparity limit" yields a percept of depth; distances greater than this limit cause the two unfused monocular features to appear flattened into the fixation plane. This behavioral disparity limit is consistent with neurophysiological estimates of the largest disparity scale in primate, allowing us to relate physiological limits on plausible binocular interactions to separation between retinal locations. Here we test the hypothesis that this upper disparity limit predicts the presence of coarse stereopsis in humans with macular degeneration (MD), which affects the central retina but typically spares the periphery. The pattern of vision loss can be highly asymmetric, such that an intact location in one eye has a corresponding point in the other eye that lies within affected retina. Nevertheless, some individuals with MD have coarse stereopsis that is useful for eye-hand coordination. Our results show that individuals with MD $(n=25$, male and female) have coarse stereopsis when the distance between intact retinal locations is less than the behavioral and physiological upper disparity limit at the corresponding eccentricity. Furthermore, for those without stereopsis, we can predict whether they can achieve stereopsis by using alternate retinal loci at further eccentricities whose separation is below the upper disparity limit.

Key words: human; macular degeneration; stereopsis; upper disparity limit

Significance Statement

We show that the largest separation between features in the two eyes that yields a percept of depth in humans is related to the largest disparity scale in macaque medial temporal area and to the estimated size of the receptive fields in human depth-sensitive cortical regions. This upper disparity limit also predicts whether individuals with retinal damage due to macular degeneration will have stereopsis. Individuals have stereopsis when the separation between intact retinal locations in the two eyes is smaller than the upper disparity limit measured behaviorally. Our results indicate the importance of the behavioral upper disparity limit as a predictor for stereopsis in populations with retinal damage.

\section{Introduction}

In primates, the lateral separation of the eyes results in each eye getting a slightly different view of an object. Disparity is the small difference in position of features in the retinal images in the two eyes and stereopsis is the percept of depth from disparity. Although there are many monocular cues to depth, stereopsis is helpful to judge the relative depth of objects, even in full-cue conditions when other monocular cues to depth are present (McKee and Taylor, 2010). While a majority of studies have focused on stereoacuity (the smallest depth differences that can be detected), studies have shown that stereopsis in general

\footnotetext{
Received Feb. 29, 2020; revised Apr. 10, 2020; accepted May 25, 2020.

Author contributions: P.V. designed research; P.V. and S.G. analyzed data; P.V. wrote the first draft of the paper; P.V. edited the paper; P.V. wrote the paper; S.G. performed research.

The authors declare no competing financial interests.

This work was supported by National Institutes of Health R01 EY-027390 to P.V. We thank Suzanne McKee

for enlightening discussions throughout the research and writing stages.

Correspondence should be addressed to Preeti Verghese at preeti@ski.org.

https://doi.org/10.1523/JNEUROSCI.0491-20.2020

Copyright ( 2020 the authors
}

improves eye-hand coordination, and even coarse stereopsis is better than none (Melmoth et al., 2009; Verghese et al., 2016). In particular, our previous study with individuals with central field loss due to macular degeneration (MD) has shown that those with residual stereopsis in the periphery are better able to place a peg in a pegboard quickly (Verghese et al., 2016). To determine the limits of stereopsis in the periphery, we measured the largest disparity that can be encoded at a given eccentricity in healthy controls (Ghahghaei et al., 2019). Here we investigate how this behavioral upper disparity limit is related to physiological measures of the scale of coarsest disparity units as a function of eccentricity.

We compared the upper disparity limit with the largest disparity range of single units as a function of eccentricity in macaque medial temporal area (MT) (DeAngelis and Uka, 2003; Parker, 2007), known to code coarse disparity (Uka and DeAngelis, 2006). We also derived an estimate of single-unit size in the human analog of MT from population receptive field (pRF) data from Amano et al. (2009) and used data from Keliris et al. (2019) to estimate the approximate conversion factor from 
pRF size to single-unit size in humans. Both estimates of the coarsest disparity scale from macaque and human are consistent with the behavioral upper disparity limit, supporting the idea that the largest disparity at a given eccentricity has a direct relationship to the coarsest scale at that eccentricity.

The correspondence between the behavioral disparity limit and neurophysiological estimates of the largest disparity scale in primate allows us to relate retinal distance to physiological limits on plausible interactions between retinal locations. The impairment of stereopsis in human MD is related directly to regions of retinal damage in either eye. MD impacts the fovea and the macula, and the pattern of vision loss in the two eyes can be highly asymmetric. Here we examine how the distance between intact retinal locations in the periphery of the two eyes is related to the upper disparity limit, and thus to the ability to discriminate differences in depth from stereopsis.

\section{Materials and Methods}

Potential physiological bases for upper disparity limit. We compared the upper disparity limit measured in Ghahghaei et al. (2019) with physiological measures of the largest disparity encoded as a function of eccentricity (DeAngelis and Uka, 2003; Parker, 2007) in macaque and humans, We chose to examine the disparity scale of neurons in area MT in primate (and its human analog $\mathrm{hMT}^{+}$) for three reasons: (1) this area appears to have the largest disparity scale among visual areas V1, V2, V4, and MT in macaque (Parker, 2007); (2) it is tuned for coarse disparities (Uka and DeAngelis, 2006); and (3) $\mathrm{hMT}^{+}$has the broadest disparity tuning of visual areas in both ventral and dorsal cortex (Preston et al., 2008). The red dashed line in Figure 1 is a smooth fit to the largest disparity scale in MT as a function of eccentricity (DeAngelis and Uka, 2003), extrapolated to the fovea $\left(0^{\circ} \mathrm{ec}-\right.$ centricity). As disparity scale is the reciprocal of disparity frequency, we have plotted half the disparity scale as the largest disparity that can be encoded by a phase-disparity mechanism, corresponding to a maximum phase shift of $\pi$ (solid red line), as larger disparities in area MT are coded by phase-disparity mechanisms (DeAngelis and Uka, 2003). We also compared the disparity limit to human pRF size (space constant $\sigma$ of the best-fitting Gaussian) from hMT ${ }^{+}$. We took the estimate of pRF size from region TO-1 from Amano et al. (2009) as this area is thought to include $\mathrm{hMT}^{+}$. As pRF size (black dashed line) overestimates the size of individual RFs due to position scatter of neurons that contribute to the population response, we used a scaling factor estimated from macaque (Keliris et al., 2019), comparing pRF sizes in V1 measured with fMRI with single-unit sizes measured with electrophysiology. Admittedly, we are assuming that $\mathrm{hMT}^{+}$in human has a similar scaling factor between $\mathrm{pRF}$ and single-unit size as does macaque V1. The scaling factor was computed as the ratio of the linear fits to the pRF and single-unit data, respectively, as a function of eccentricity. These values were taken from Keliris et al. $(2019$, their Table S2): pRF size $=1.39+0.19 e$, single-unit size $=0.16+0.08 e$, where $e$ is the eccentricity in degrees. We scaled the human pRF data from Amano et al. (2009) by the scaling factor to estimate the size of single units in human $\mathrm{MT}^{+}$. As size and disparity are correlated (Marr and Poggio, 1979; Schor et al., 1984; Prince et al., 2002), and monocular RF components of disparity units are known to overlap, we assume that the largest disparity is equal to the full width at half height (W) of the single-unit RF at that eccentricity, and used the expression $\mathrm{W}=2.36 \sigma$ to estimate full width in $\mathrm{hMT}^{+}$. The solid black line in Figure 1 estimates the full width of a single neuron as a function of eccentricity.

Comparison of behavioral and physiological disparity limit with retinal separations in $M D$. Our study included 25 human participants with macular disease (13 females), 52-92 years of age who were referred to us by Donald Fletcher (Low Vision Rehabilitation, California Pacific Medical Center, San Francisco) (Table 1). Participants had the agerelated form of the disease $(n=18)$, the juvenile form of the disease $(n=4)$, or other maculopathies $(n=3)$. All participants provided written consent before participation in our study. The study protocol was approved by the Institutional Review Board at the Smith-Kettlewell Eye

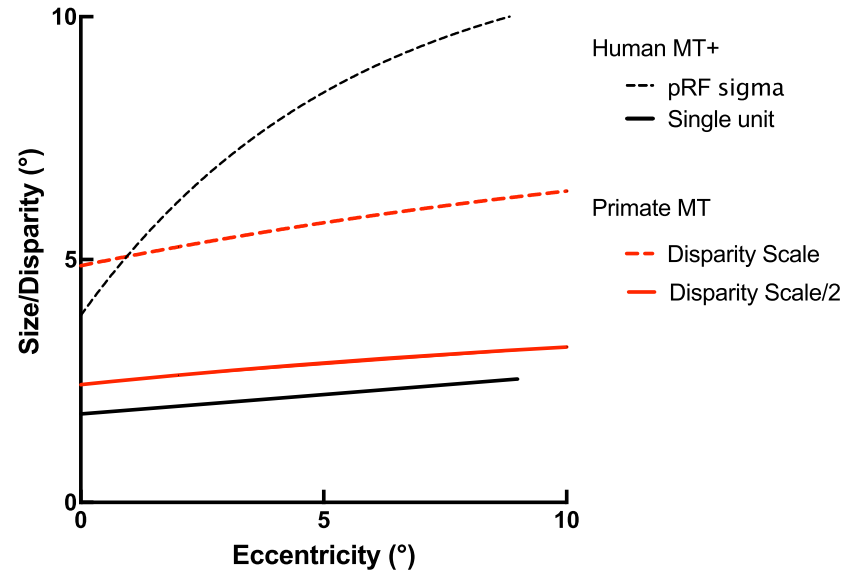

Figure 1. Physiologic estimates of the upper disparity limit as a function of eccentricity. Black lines indicate data of human RF size in human $\mathrm{MT}^{+}$as a function of eccentricity. Dashed line indicates space constant of pRF from Amano et al. (2009). Solid line indicates single-unit size derived from pRF, scaled by factor from Keliris et al. (2019). Red lines indicate the largest disparity units in macaque MT from DeAngelis and Uka (2003). Dashed line indicates disparity scale. Solid line indicates the largest disparity encoded.

Table 1. Study participants with their monocular acuities, PRL eccentricity, and whether or not they have measurable stereopsis ${ }^{a}$

\begin{tabular}{lllllc}
\hline Patient & $\begin{array}{l}\text { Age } \\
\text { ID }\end{array}$ & $\begin{array}{l}\text { OS acuity } \\
\text { (logMAR) }\end{array}$ & $\begin{array}{l}\text { OS acuity } \\
\text { (logMAR) }\end{array}$ & $\begin{array}{l}\text { OS PRL } \\
\text { eccentricity }\left(^{\circ}\right)\end{array}$ & $\begin{array}{c}\text { OD PRL } \\
\text { eccentricity }\left({ }^{\circ}\right)\end{array}$ \\
\hline S1 & 73 & 1.1 & 0.8 & 8.2 & 7.7 \\
S2 & 76 & 1.1 & 1.2 & 14.3 & 14.3 \\
S3 & 80 & 0.4 & 0.1 & 3.2 & 0.0 \\
S4 & 83 & 0.98 & 1.2 & 1.4 & 8.6 \\
S5 & 90 & 0.0 & 0.3 & 0.0 & 1.0 \\
S6 & 74 & 0.1 & 0.4 & 0.0 & 2.8 \\
S7 & 76 & 0.55 & 0.87 & 2.0 & 3.7 \\
S8 & 58 & 1.1 & 1.1 & 5.2 & 3.8 \\
S9 & 70 & 1.1 & 0.1 & 4.8 & 0.4 \\
S10 & 76 & 0.6 & 1.24 & 7.8 & 7.9 \\
S11 & 72 & 0.6 & 0.73 & 1.1 & 2.2 \\
S12 & 78 & 0.3 & 0.41 & 0.0 & 2.0 \\
S13 & 87 & 1.1 & 0.56 & 5.7 & 6.5 \\
S14 & 68 & 0.6 & 1.85 & 1.7 & 8.1 \\
S15 & 85 & 1.2 & 0.94 & 8.5 & 6.9 \\
S16 & 77 & 0.81 & 0.3 & 2.9 & 1.6 \\
S17 & 80 & 0.4 & 0.4 & 2.0 & 0.0 \\
NS1 & 87 & 1 & 0.7 & 1.3 & 2.3 \\
NS2 & 52 & 0.57 & 1.5 & 12.3 & 10.9 \\
NS3 & 94 & 0.3 & 1.4 & 2.2 & 5.5 \\
NS4 & 56 & 1.12 & 1.67 & 1.5 & 7.9 \\
NS5 & 87 & 0.6 & 0.6 & 2.3 & 4.0 \\
NS6 & 92 & 0.1 & 1.5 & 2.5 & 5.2 \\
NS7 & 58 & 1.34 & - & 10.7 & 11.9 \\
NS8 & 90 & 1.15 & 1.38 & 4.2 & 5.9 \\
\hline
\end{tabular}

${ }^{\mathrm{a}} \mathrm{OS}$, Left eye; OD, right eye; $\mathrm{S}$, stereopsis; NS, no measurable stereopsis.

Research Institute and conformed to the guidelines of the Declaration of Helsinki for the treatment of human subjects.

We performed monocular microperimetry in each eye using the Optos optical coherence tomograph/scanning laser ophthalmoscope, with a field size of $29.7^{\circ}$. We used a custom arrangement of points to determine the scotoma profile up to about an eccentricity of $15^{\circ}$. Scotoma mapping was performed with unattenuated $0 \mathrm{~dB}$ dots (dot luminance $125 \mathrm{~cd} / \mathrm{m}^{2}$; Weber contrast 12.5). Fixation stability and the preferred retinal locus (PRL) for static fixation were also measured monocularly, using a $10 \mathrm{~s}$ fixation target. The location of the foveal pit was determined using the radial scan function of the optical coherence 

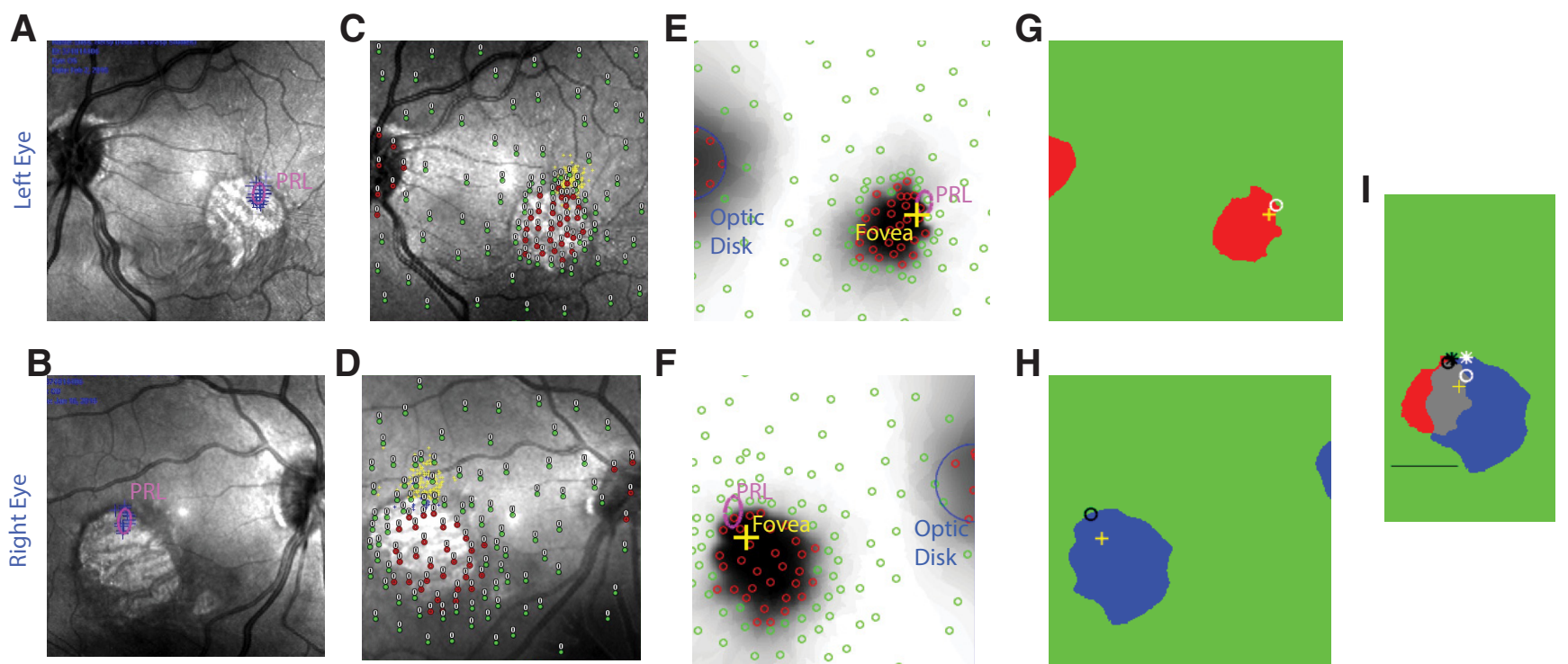

D

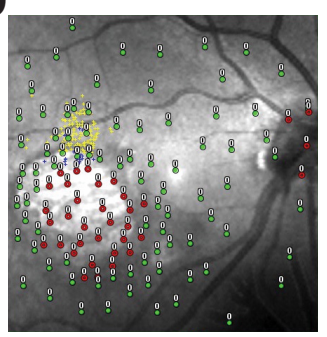

$\mathbf{F}$

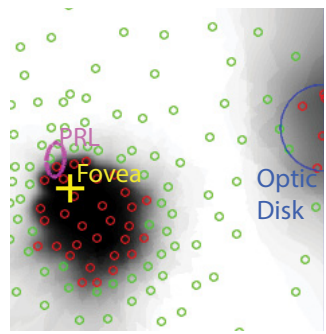

H

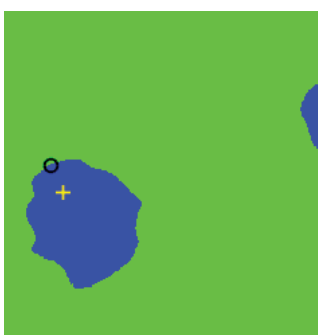

Figure 2. Evaluating the potential for stereopsis in 25 individuals with MD by estimating the binocular overlap of the monocular scotomata. $\boldsymbol{A}, \boldsymbol{B}$, Scanning laser ophthalmoscope images of left and right eyes, respectively, for Participant S11. Blue crosses represent fixation loci. Pink oval represents the two-dimensional Gaussian fit to the fixation positions, and marks the PRL. $\boldsymbol{C}$, $\boldsymbol{D}$, Scanning laser ophthalmoscope images for left and right eyes with microperimetry data overlapped. Green and red dots represent seen and missed flashes, respectively. $\boldsymbol{E}$, $\boldsymbol{F}$, Smoothed scotoma profiles in each eye with the optic disk, fovea, and PRL marked. $\boldsymbol{G}, \boldsymbol{H}$, Thresholded maps for each eye. $\boldsymbol{I}$, The two eyes' maps overlapped and aligned on the respective foveae (yellow cross). Red, blue, and gray represent the scotoma profiles of the left and right eyes, and the region of binocular overlap, respectively. The distance from the PRL (circle) in one eye to the nearest intact location in the other eye (asterisk) is depicted as the separation between symbols of the same color. Black horizontal line indicates a scale marker of $5^{\circ}$. The overlapped map is narrower because it only includes areas of binocular overlap.

tomograph. In cases where we could not visualize the foveal pit in one eye because of disease progression, we took advantage of the finding that the two eyes have very similar locations of the fovea with respect to the center of the optic disk (Rohrschneider, 2004) and used the other eye's measurement to estimate this distance. For one individual (Participant S17) for whom we could not visualize the foveal pit in either eye due to macular edema, we used normative data from a control population to determine the fovea location from the center of the optic disk, $15^{\circ}$ horizontally and 1.5 degree vertically below (Hu et al., 1994; Kabanarou et al., 2006). To determine the overlap of the scotomata in the two eyes, we aligned the perimetry profiles of each eye on the foveal pits as in Verghese et al. (2016) and demonstrated in Figure 2.

We determined the extent of the scotoma in each eye, the PRL, and the location of the foveal pit in each eye as described previously (Ghahghaei and Walker, 2016; Verghese et al., 2016). We assumed that our participants' eyes were aligned during binocular viewing, as most individuals with MD appear to align their gaze with the PRL in the dominant eye when viewing binocularly (Kabanarou et al., 2006; Tarita-Nistor et al., 2012). This assumption allowed us to superimpose the retinal maps of the two eyes, aligned on the foveae, to estimate locations capable of mediating stereopsis (Fig. $2 A-I$ ). The PRL in each eye is typically close to the edge of the scotoma, often in a region that yields close to the best acuity. We measured the distance between this PRL and the nearest intact retinal location in the other eye and compared this distance with the upper disparity limit measured psychophysically (Ghahghaei et al., 2019) as well as to physiological measures of the largest disparity in middle temporal complex (MT), which codes depth from disparity.

Stereopsis was measured using the Random dot stereo butterfly test, which measures stereopsis up to $2000 \mathrm{arc} \mathrm{sec}$. Because there are monocular cues in the Randot circles and animals (Chopin et al., 2019), observers needed to perceive depth in the Randot butterfly to qualify as having real stereopsis.

Statistics. We calculated means and 95\% CIs for retinal distance between intact locations in the two eyes as well as for eccentricity of the PRL in the dominant eye, for groups with and without measurable stereopsis.

\section{Results}

\section{Relating physiological and behavioral estimates of the upper disparity limit}

Before we compared the distance between intact retinal locations in the two eyes of patients with MD to the upper disparity limit, we investigated potential physiological bases for this limit. As area MT in macaque is tuned for coarse disparities (Uka and DeAngelis, 2006), and its human analog $\mathrm{hMT}^{+}$has the broadest disparity tuning of visual areas in both ventral and dorsal cortex (Preston et al., 2008), we chose this cortical area as a likely candidate to mediate sensitivity to large disparities. We used the largest disparity scale measured across a population of MT neurons with RFs that spanned eccentricities from $2^{\circ}$ to $20^{\circ}$ (DeAngelis and Uka, 2003) and then scaled these values down by a factor of 2 , assuming that the largest disparity that can be encoded by a phase-disparity mechanism corresponds to a maximum phase shift of $\pi$ (see Materials and Methods). The red line in Figure 3 shows our estimates of the largest disparities coded in macaque MT as a function of eccentricity.

For comparison with human data, we considered human $\mathrm{pRF}$ size from $\mathrm{hMT}^{+}$(Amano et al., 2009), and used a scaling factor from Keliris et al. (2019) to estimate the size of single neurons for pRFs (see Materials and Methods). As size and disparity are correlated (Marr and Poggio, 1979; Schor et al., 1984; Prince et al., 2002), and monocular RF components of disparity units are known to overlap, we assume that the largest disparity is equal to the full width at half height (W) of the single-unit RF at that eccentricity. The solid black line in Figure 3 plots the estimate of $\mathrm{RF}$ size in $\mathrm{hMT}^{+}$as a function of eccentricity. The orange symbols plot the average upper disparity limit as a function of eccentricity for 7 control participants from Ghahghaei et al. (2019). The error bars indicate $95 \%$ CIs of the mean. Remarkably, the physiological estimates of disparity scale in macaque MT and single-unit RF size in human $\mathrm{MT}^{+}$are comparable to the 
behavioral estimate of the upper disparity limit in humans (Ghahghaei et al., 2019). In other words, inputs from the two eyes are coded by RFs that overlap in the two eyes. If the images in the two eyes have a disparity greater than this spacing, then they do not combine binocularly corresponding regions and cannot encode depth.

\section{Comparison of upper disparity limit with retinal separation between intact locations in MD}

How do the upper disparity limit data relate to the presence or absence of stereopsis in individuals with MD? Our prediction is that the upper disparity limit and the RF size at a given eccentricity set an upper bound on the separation between intact retinal locations that can support stereopsis in individuals with central field loss. As a reference point, we consider the PRL in the dominant eye and calculate the distance to intact retina in the other eye.

Our results from 25 individuals with central field loss due to MD are shown in Figure 4, which plots the separation between the dominant-eye PRL and healthy retina in the other eye versus the eccentricity of the dominant-eye PRL. Figure $4 A$ also replots the retinal separation versus eccentricity data, along with the behavioral upper disparity limit that we measured previously in normally sighted control observers (Ghahghaei et al., 2019). It is clear that each individual with MD with stereopsis ( $n=17$; open circles) has a distance between healthy retinae in the two eyes that is at or below the upper disparity limit. The upper disparity limit is consistent with the physiological upper bound of the largest RF separation that can encode disparity. Thus, our results show that it is the separation between intact retinal locations that determines stereopsis.

Figure $4 B$ partitions this interocular retinal distance into its horizontal and vertical components to determine the relation of the horizontal component of this separation to the upper disparity limit measured in normally sighted controls (Ghahghaei et al., 2019). The upper disparity limit grows slowly with eccentricity from $\sim 2^{\circ}$ at the fovea to $3.6^{\circ}$ at an eccentricity of $10^{\circ}$. Despite ignoring the small effect of eccentricity on the upper disparity limit, participants with measurable stereopsis have points clustered around the origin (open symbols), with horizontal and vertical separations $\leq 1.6^{\circ}$ (1 participant has a horizontal separation of $2.5^{\circ}$, which is within the range of the upper disparity limit that we measured). Participants without stereopsis (filled symbols) have larger values of horizontal and vertical separations. These results are consistent with the assumption that the vertical extent of the binocular integration zone (Parker et al., 2016) is similar to the scale for horizontal disparity, consistent with the results from physiology in macaque V1 (Nienborg et al., 2004).

Another potential marker for stereopsis is the interocular separation between the monocular PRLs. Figure 5 shows that the relation of this distance to the upper disparity limit is much less systematic, with individual interocular PRL distances both above and below this limit. The upper disparity limit does not separate even the mean data for the groups with and without stereopsis.

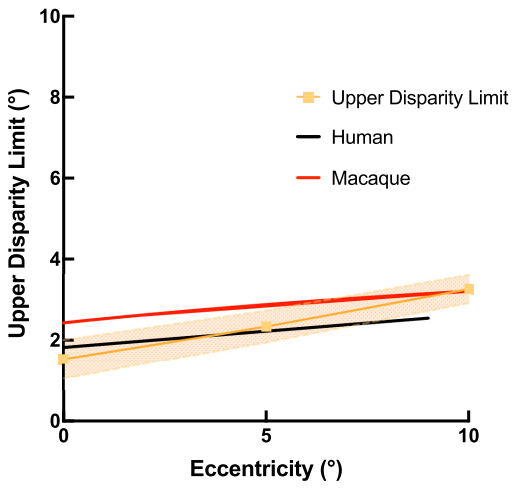

Figure 3. Relation between behavioral and physiological estimates of the upper disparity limit. Orange symbols represent the upper disparity limit from [1] and along with the 95\% confidence limits of the mean. Black line indicates estimated single-unit size as a function of eccentricity in human $\mathrm{MT}^{+}$, replotted from Figure 1. Red line indicates the estimate of the largest disparity encoded in macaque MT from DeAngelis and Uka (2003), also replotted from Figure 1.
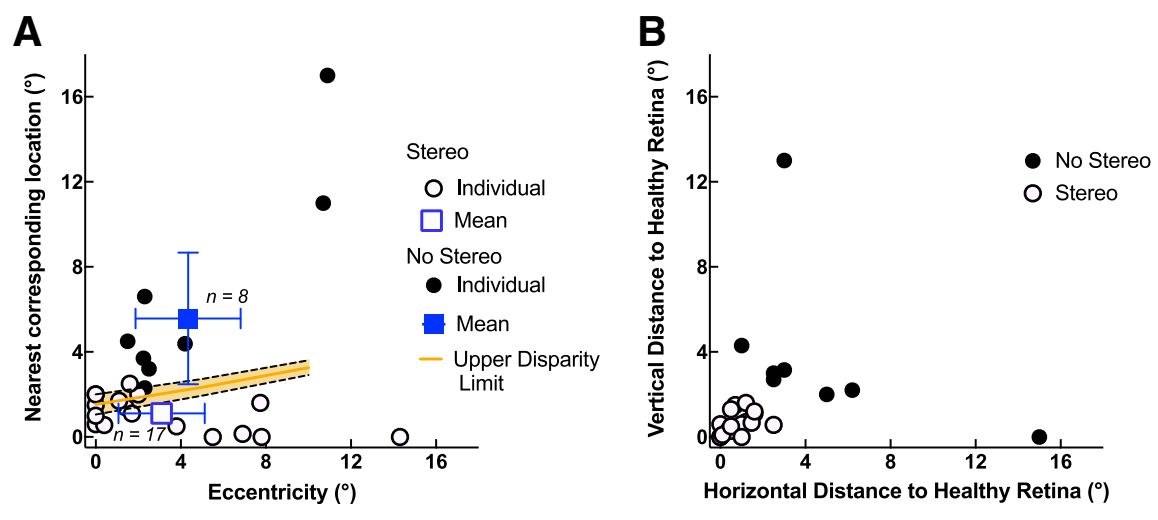

Figure 4. Separation between healthy locations in the two eyes. $\boldsymbol{A}$, The distance between dominant-eye PRL and the nearest intact location in the other eye is plotted as a function of PRL eccentricity. Open and filled circles represent data for individuals with and without measurable stereopsis, respectively. Corresponding blue squares represent mean data for the two groups. Error bars indicate $95 \% \mathrm{Cls}$ (he stereopsis have data points that lie largely above the upper disparity limit. $\boldsymbol{B}$. The vertical versus horizontal components of the separation between the dominant eye PRL and the nearest intact location in the other eye, ignoring the eccentricity of the dominant-eye PRL. Open and filled circles represent data for individuals with and without measurable stereopsis, respectively.

This is not surprising as the monocular PRLs are determined by each eye's scotoma profile, which can be highly asymmetric between the two eyes. Binocular viewing typically uses the dominant eye PRL (Kabanarou et al., 2006; Tarita-Nistor et al., 2012), with the nondominant eye changing its gaze to be roughly aligned with the dominant eye.

\section{Discussion}

We show that the behavioral upper disparity limit is consistent with physiological estimates of the largest disparity encoded by disparity-sensitive neurons as a function of eccentricity. This is true for the coarsest disparity scale as a function of eccentricity in macaque MT, measured directly. We estimated RF size in human $\mathrm{MT}^{+}$by scaling down the measured pRF values (Amano et al., 2009) in humans by a scale factor determined by comparing $\mathrm{pRF}$ and physiological estimates of single-unit size in macaque (Keliris et al., 2019). We assume that the estimate of RF size at a given eccentricity is the physiological upper bound of the largest RF separation that can encode disparity. In other words, 


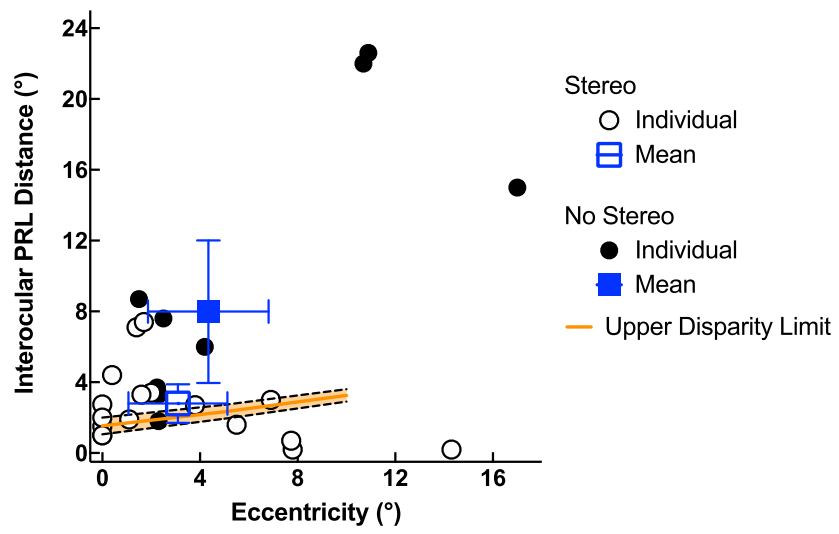

Figure 5. The separation between the PRL loci in the two eyes is plotted as a function of the dominant-eye PRL eccentricity. Open and filled circles represent data for individuals with and without measurable stereopsis, respectively. Corresponding squares represent mean data for the two groups. Error bars indicate $95 \%$ Cls of the mean. Orange represents the upper disparity limit; it does not cleanly divide participants into groups with and without stereopsis.

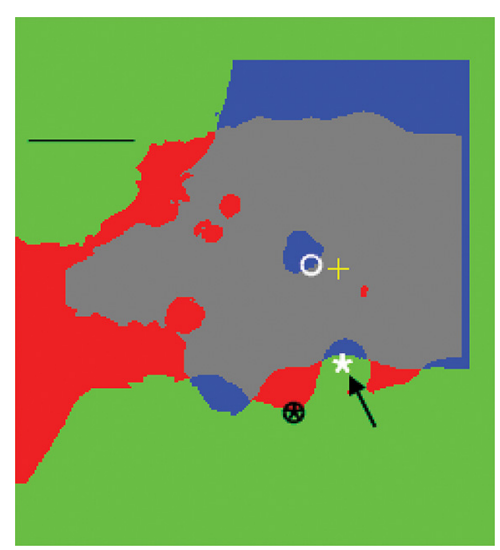

Figure 6. Binocular scotoma map for Participant NS4. Yellow cross represents the fovea. Red and blue represent the left and right eye scotomas, respectively. The distance from the PRL (circle) in one eye to the nearest intact location in the other eye (asterisk) is depicted as the separation between symbols of the same color. Black horizontal line indicates a scale marker of $5^{\circ}$. This participant has near-foveal sparing in the left eye within a large scotoma in the right eye, and no measurable stereopsis. Black arrow indicates an alternate fixation locus that is intact in both eyes and could provide stereopsis.

inputs from the two eyes are coded by RFs that overlap in the two eyes. This physiological correlate allows us to map separation between retinal locations to the size of the coarsest scale of units coding stereopsis, and to predict whether individuals with MD will have stereopsis depending on the distance between healthy retina in the two eyes.

We chose to compare our results with area MT rather than another visual cortical area for several reasons. We were interested in the largest retinal separation between image features that supports coarse stereopsis. Although the upper disparity ranges of neurons in V2 and MT are quite similar (Cumming and DeAngelis, 2001), we chose to compare our human behavioral data with the disparity range in MT because there is evidence that MT mediates perceptual decisions about coarse disparity in macaque. Uka and DeAngelis (2006) showed that micro-stimulation of MT affected perceptual sensitivity in a coarse disparity discrimination task but not in a fine task. This is in contrast to disparity-selective neurons in V1, whose properties are not consistent with behavioral disparity sensitivity. For instance, V1 neurons are sensitive to the local disparity of a grating within their RF, and not sensitive to the disparity of edges that determine the percept of depth (Cumming and Parker, 2000). V1 neurons also respond to the disparity of anticorrelated dots, which do not elicit a consistent depth percept (Cumming and Parker, 1997). Furthermore, V1 neurons appear to code absolute and not relative disparity, which is essential for everyday tasks requiring disparity differences (Cumming and Parker, 1999).

We acknowledge that we have made various assumptions to estimate disparity scale. For human data, we know of no direct measure of the coarsest disparity scale in $\mathrm{hMT}^{+}$, so we have assumed that the size-disparity relation reported in macaque $\mathrm{V} 1$ (Prince et al., 2002) also applies to area MT, and use RF size to estimate the largest disparity scale. Similarly, there are no reports that relate the extent over which a single unit integrates disparity in macaque MT (the binocular integration zone; Parker et al., 2016) to the size of the RF, unlike macaque V1. For V1, Nienborg et al. (2004) used a stimulus corrugated in depth to show a close relation between the binocular integration zone and RF size in macaque V1. If we assume that a similar relation exists for neurons in macaque MT, and by analogy in human $\mathrm{hMT}^{+}$, we can relate the binocular integration zone to RF size.

For 25 participants with MD, the upper disparity limit clearly separates those with and without stereopsis. The relationship between the presence of stereopsis and the distance between spared retina in the two eyes applies across a heterogeneous pattern of vision loss in our sample, ranging from small monocular to large binocular scotomata, and from compact to patchy vision loss. The behavioral and physiological upper disparity limit consistently divides this population into those with and without stereopsis, with only one exception: an individual with separation between intact retinal locations at this limit, but no measurable stereopsis (Participant NS5). This individual has large binocular scotomata, with small islands of intact function that are likely too small to mediate reliable stereopsis.

\section{Potential for rehabilitation}

Our method also allows us to predict whether individuals without measurable stereopsis have the potential for stereopsis in their periphery at locations remote from their dominant-eye PRL. Many individuals with MD, particularly those with spared retinal function near the fovea in one eye and a large central scotoma in the other eye, often rely on the eye with high acuity and ignore peripheral retina that is healthy in both eyes, which can mediate coarse stereopsis. Not surprisingly, they often complain that they are clumsy when they reach for objects in real life. Our mapping procedure can predict which peripheral loci are capable of stereopsis. For example, Participant NS4 has large scotomata in the two eyes with near-foveal sparing in the left eye (Fig. 6): the PRL in the left eye is near the fovea in a region with an extensive scotoma in the right eye. Predictably, this participant has no measurable stereopsis. However, he has intact retina in both eyes at a distance of $\sim 4.5^{\circ}$ from the fovea. Using this more peripheral location as an alternate PRL is likely to provide depth from disparity. Previous studies have shown that individuals with MD learn to use different PRLs depending on the task (Whittaker et al., 1988; Guez et al., 1993; Sunness et al., 1996; Lei and Schuchard, 1997; Déruaz et al., 2002). Thus, it is possible to train awareness of retinal loci that can mediate stereopsis.

In conclusion, our method to map the residual visual function in nearly corresponding parts of the two eyes predicts which individuals have the capacity for peripheral stereopsis, a function 
that has significant impact on tasks of daily living, such as eyehand coordination and navigation (Cao and Markowitz, 2014; Verghese et al., 2016). Furthermore, our method has the potential to identify alternate fixation loci that can provide depth from disparity.

\section{References}

Amano K, Wandell BA, Dumoulin SO (2009) Visual field maps, population receptive field sizes, and visual field coverage in the human MT+ complex. J Neurophysiol 102:2704-2718.

Cao KY, Markowitz SN (2014) Residual stereopsis in age-related macular degeneration and its impact on vision-related abilities: a pilot study. J Optom 7:100-105.

Chopin A, Chan SW, Guellai B, Bavelier D, Levi DM (2019) Binocular and non-stereoscopic cues can deceive clinical tests of stereopsis. Sci Rep 9:5789.

Cumming BG, DeAngelis GC (2001) The physiology of stereopsis. Annu Rev Neurosci 24:203-238.

Cumming BG, Parker AJ (1997) Responses of primary visual cortical neurons to binocular disparity without depth perception. Nature 389:280-283.

Cumming BG, Parker AJ (1999) Binocular neurons in V1 of awake monkeys are selective for absolute, not relative, disparity. J Neurosci 19:5602-5618.

Cumming BG, Parker AJ (2000) Local disparity not perceived depth is signaled by binocular neurons in cortical area V1 of the Macaque. J Neurosci 20:4758-4767.

DeAngelis GC, Uka T (2003) Coding of horizontal disparity and velocity by MT neurons in the alert macaque. J Neurophysiol 89:1094-1111.

Déruaz A, Whatham AR, Mermoud C, Safran AB (2002) Reading with multiple preferred retinal loci: implications for training a more efficient reading strategy. Vision Res 42:2947-2957.

Ghahghaei S, McKee SP, Verghese P (2019) The upper disparity limit increases gradually with eccentricity. J Vis 19:3.

Ghahghaei S, Walker L (2016) SKERI-Optos: a graphical user interface to map scotoma and PRL with the Optos OCT/SLO. J Vis 16:40-41.

Guez J-E, Le Gargasson J-F, Rigaudiere F, O’Regan JK (1993) Is there a systematic location for the pseudo-fovea in patients with central scotoma?. Vision Research 33:1271-1279.

Hu S, Schuchard R, Fletcher D, Sabates F (1994) Physiological blind spot characteristics and position relative to retinal locus for fixation by SLO testing. Invest Ophthalmol Vis Sci 35:1527.

Kabanarou SA, Crossland MD, Bellmann C, Rees A, Culham LE, Rubin GS (2006) Gaze changes with binocular versus monocular viewing in agerelated macular degeneration. Ophthalmology 113:2251-2258.
Keliris GA, Li Q, Papanikolaou A, Logothetis NK, Smirnakis SM (2019) Estimating average single-neuron visual receptive field sizes by fMRI. Proc Natl Acad Sci USA116:6425-6434.

Lei H, Schuchard RA (1997) Using two preferred retinal loci for different lighting conditions in patients with central scotomas. Invest Ophthalmol Vis Sci 38:1812-1818.

Marr D, Poggio T (1979) A computational theory of human stereo vision. Proc R Soc Lond B Biol Sci 204:301-328.

Melmoth DR, Finlay AL, Morgan MJ, Grant S (2009) Grasping deficits and adaptations in adults with stereo vision losses. Invest Ophthalmol Vis Sci 50:3711-3720

McKee SP, Taylor DG (2010) The precision of binocular and monocular depth judgments in natural settings. J Vis 10:5.

Nienborg H, Bridge H, Parker AJ, Cumming BJ (2004) Receptive field size in V1 neurons limits acuity for perceiving disparity modulation. J Neurosci 24:2065-2076.

Parker AJ (2007) Binocular depth perception and the cerebral cortex. Nat Rev Neurosci 8:379-391.

Parker AJ, Smith JE, Krug K (2016) Neural architecture for stereo vision. Philos Trans R Soc Lond B Biol Sci 19:371.

Preston TJ, Li S, Kourtzi Z, Welchman AE (2008) Multivoxel pattern selectivity for perceptually relevant binocular disparities in the human brain. J Neurosci 28:11315-11327.

Prince SJ, Cumming BG, Parker AJ (2002) Range and mechanism for encoding horizontal disparity in macaque V1. J Neurophysiol 87:209-221.

Rohrschneider K (2004) Determination of the location of the fovea on the fundus. Invest Ophthalmol Vis Sci 45:3257-3258.

Schor C, Wood I, Ogawa J (1984) Binocular sensory fusion is limited by spatial resolution. Vision Res 24:661-665.

Sunness JS, Applegate CA, Haselwood D, Rubin GS (1996) Fixation patterns and reading rates in eyes with central scotomas from advanced atrophic age-related macular degeneration and Stargadt's disease. Ophthalmology 103:1458-1466

Tarita-Nistor L, Brent MH, Steinbach MJ, González EG (2012) Fixation patterns in maculopathy: from binocular to monocular viewing. Optom Vis Sci 89:277-287.

Uka T, DeAngelis GC (2006) Linking neural representation to function in stereoscopic depth perception: roles of the middle temporal area in coarse versus fine disparity discrimination. J Neurosci 26:6791-6802.

Verghese P, Tyson TL, Ghahghaei S, Fletcher DC (2016) Depth perception and grasp in central field loss. Invest Ophthalmol Vis Sci 57:1476-1487.

Whittaker SG, Budd J, Cummings RW (1988) Eccentric fixation with macular scotoma. Invest Ophthalmol Vis Sci 29:268-278. 